\title{
Plattform V „immobil - Ort für Wirklichkeitssprünge“
}

Eric Wagner, Projektleiter Plattform V

In Kooperation mit der Kunsthalle Lingen und der Fachhochschule Osnabrück, Standort Lingen (Ems) / Institut für Theaterpädagogik, jenseits der Bahn, September 2006

\section{Eine ästhetische Operation ${ }^{1}$ - Rückblick}

Der Ausgangspunkt für die Idee der Plattform „immobil“ fand sich in der Auseinandersetzung mit der Begrifflichkeit „Tunnelblick“. Der Begriff zeigt auf, dass die Selektion der Wahrnehmung zur Betrachtung avanciert. Der Titel beschreibt ein „nicht offen sein“ für die Vielfalt an Sinneseindrücken, blendet das auditive Erleben aus und reduziert sich bis zu einem negativen Bild der Enge. In der Etymologie des Wortes „Tunnel“ finden wir jedoch auch den Verweis auf Fass, Tonne oder Schacht. In dem Roman von Haruki Murakami ${ }^{2}$ Mr. Aufziehvogel wird diese Verbindung aufgegriffen, indem er seiner Figur in einem Brunnenschacht Raum für Grenzerfahrungen, Wirklichkeitssprünge und Rückzug bietet.

Die Plattform „immobil“ schloss an dieses Motiv an und griff zusätzlich die Hypothese auf, dass Einschränkungen in der Bewegung einen sinnwandelnden „Tunnelblick“ ermöglichen. Gestaltet wurde eine performative Installation, bestehend aus zwei Schächten, die sich aus jeweils drei übereinander aufgebauten Metallsegmenten zusammensetzten, auf deren Ebenen die PerformerInnen über den Zeitraum von 12 Stunden verweilten. Im Vorfeld wurden die AkteurInnen in einem interdisziplinären Workshop vom 10. bis 14. September 2006, der Elemente aus den Bereichen bildende Kunst, Objektbau, Performance, Tanz, Theater, Soundgestaltung verband, auf diese Erfahrung vorbereitet, wobei die

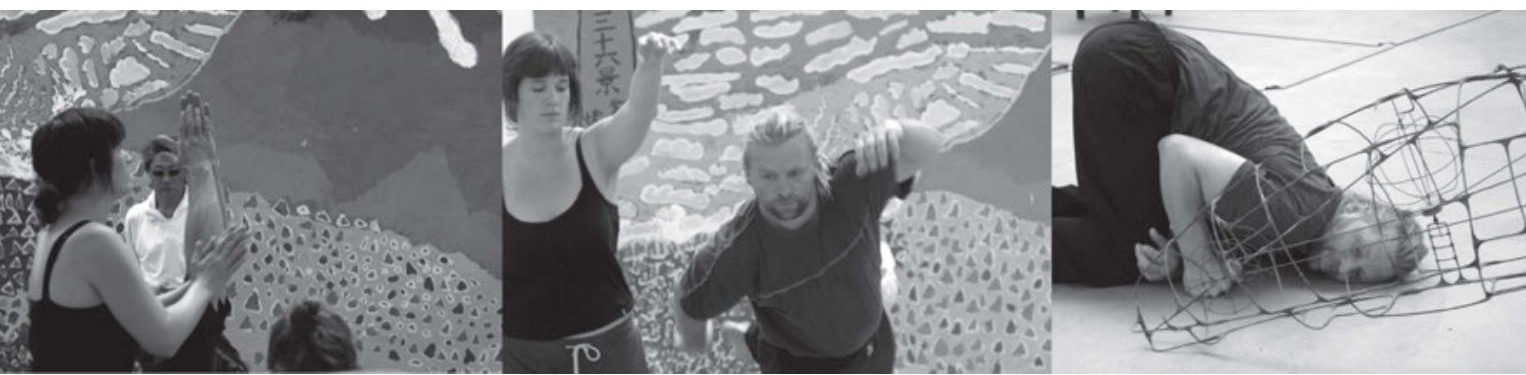

Plattform V, Fotografien: Reinhard Prüllage 
Annäherung an eine Kultur des Unfertigen, Unvollkommenen, des in Veränderung Begriffenen die Arbeit begleitete. Nicht-Perfektion gewann an Bedeutung und bot die Grundlage für ein gesteigertes intuitives Erfassen. Die Gegenwartsorientierung und die Vertrautheit mit Uneindeutigkeit und bestehenden Widersprüchlichkeiten des Konzeptes fand sich in den Fragestellungen der AkteurInnen und Beteiligten. Fragen, die sich mit der eigenen psychophysischen Belastbarkeit auseinander setzten, aber auch Fragen, die den Ortsbezug, das städtische Tunnelbauprojekt aufgriffen (Bequemlichkeit, Sicherheit, Überwachung), angeregt u.a. von dem Projekt „1984 - Termitenstaat“ aus den Jahren 1978/79 von Harry Kramer, Künstler und Professor an der Gesamthochschule Kassel, geboren 1925, gestorben 1997.

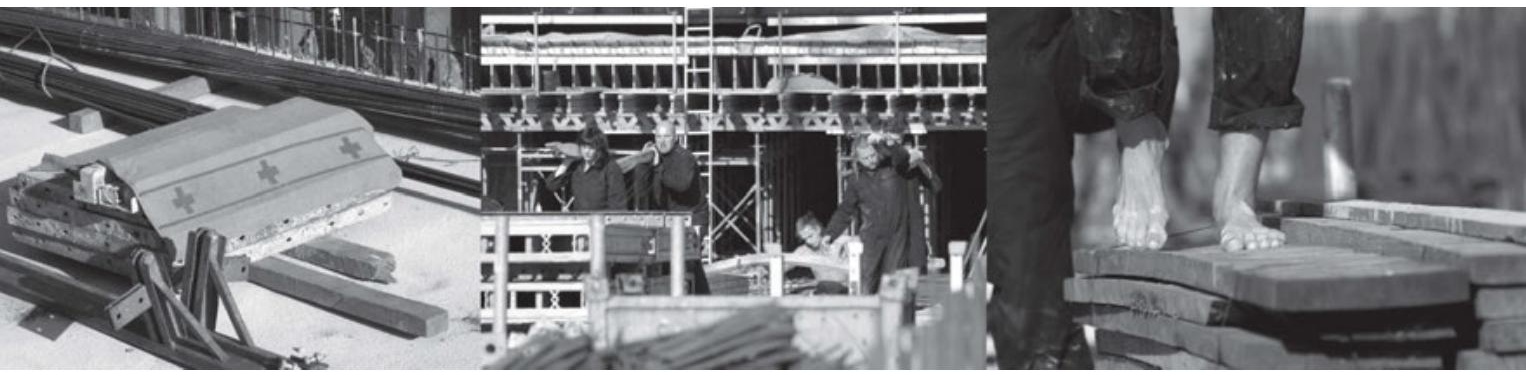

Kazuo Ono (Mitbegründer des japanischen Ausdruckstanzes Butoh) beschreibt die „Ratlosigkeit“ als seine Technik, die kein endgültiges Ergebnis als Ziel kennt. Er formuliert, dass das „Verstehen“ der Bewegung entspringen muss. Mit diesem Hintergrund wurde in dem Workshop das Augenmerk auf Bewegung gerichtet, und dabei stand der Intimste unserer Sinne, der kinästhetische Sinn, im Mittelpunkt. Tanz, insbesondere der japanische Ausdruckstanz „Butoh“, ermöglichte einen wirkungsvollen Zugang. Der bewegliche Körper wurde zum Mittel, um Erfahrungen des Geistes aus der äußeren Welt und die der Seele aus der inneren Welt (Aufspüren innerer Bilder) zum Ausdruck zu bringen, und um sich den Komponenten von Raum und Zeit in der Performance zu nähern. Tadashi Endo (Butoh-Tänzer und -Choreograph) übernahm zudem eine spezielle Workshopeinheit, wobei die Aufmerksamkeit auf den Prozess der Wandlung des Körpers lag. Ein besonderes Ohrenmerk während des Workshops wurde auf akustische Kulissen gelegt, wie z.B. der Einfluss der Kakophonien der Tunnelbaustelle, aber auch der geräuschvollen automobilen Skulpturen von Harry Kramer. Sie trugen zu der Auseinandersetzung mit dem Außen - der Umwelt - bei, bevor sich die TeilnehmerInnen für die Zeit der Performance auf ein Innen - den Schacht - zurückzogen. Für die Performerlnnen kam die Welt in den Schacht durch Blicköffnungen und durch das auditiv Wahrgenommene. Tonflächen ermöglichten eine Einflussnahme auf die Hörumwelt der Performerlnnen, PassantInnen und BesucherInnen. Gezielte Fremd- und Eigenklänge wirkten auf das Innen sowie 
das Außen und fanden vielfältige Resonanzen. Die Plattform präsentierte sich am 15. September 2006 in der Stadt Lingen, vor der Tunnelbaustelle, von morgens 10 Uhr bis abends 22 Uhr. Der Erlebnisgehalt in seiner Komprimiertheit wirkte einem „konsum-inszenatorischen Dekorationsbau“3 entgegen, wobei die Beständigkeit, die Zeitdauer viele PassantInnen und BesucherInnen zu einem verweilenden, wiederkehrenden Anschauen und Teilhaben veranlasste, was den Gewohnheiten des Publikums in der Regel nicht entspricht. Aus diesem Grund kann auch auf Seiten der RezipientInnen von einer neuen Erfahrung gesprochen werden. Die Schächte wurden während der Performance für kürzere Zeitabschnitte geöffnet. Durch die Begehbarkeit nahmen die BesucherInnen, insbesondere Kinder, von der Installation Besitz, erforschten im Spiel die sich bietenden Möglichkeiten der Interaktion, das Innen und Außen, und schufen somit die „Schnittstelle Kunst - Vermittlung“. Die Subjektivität, Individuation und Intimität der PerformerInnen und der RezipientInnen sowie die Intensität, die Größe und der Charakter des Wirkungsraumes und seiner Fraktale ließen Sprünge in eine eigentlich konkrete Wirklichkeit zu. Sprünge, die der von Christian Morgner (Soziologe) beschriebenen „Bewegung“ des „Unsinn“4 entsprechen.

\section{Anmerkungen}

1 Mit diesem Begriff bezieht sich Eric Wagner nicht auf Pierangelo Maset, der inn in seiner Publikation Maset, Pierangelo (2001): Praxis Kunst Pädagogik. Ästhetische Operationen in der Kunstvermittlung. Lüneburg: edition Hyde verwendet. Stattdessen geht es ihm bei der Verwendung um das Assoziationsfeld des medizinischen Eingriffs, insbesondere der Chirurgie.

2 Murakami, Haruki (2000): Mr. Aufziehvogel. München: btb.

3 Schepers, Heiner (Hg.) (1997): Ein Stunde für Harry Kramer. Lingen: Buxus.

4 Christian Morgner in einem unveröffentlichten Vortrag im Rahmen des Symposions DAGA Unsinn schafft Bewegung. Friedrichshof/Kublank. 2005.

\section{Literatur}

Maset, Pierangelo (2001): Praxis Kunst Pädagogik. Ästhetische Operationen in der Kunstvermittlung. Lüneburg: edition Hyde verwendet.

Schepers, Heiner (Hg.) (1997): Ein Stunde für Harry Kramer. Lingen: Buxus.
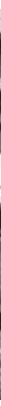